\title{
On Fuzzy Bi-Level Multi-Objective Large Scale Integer Quadratic Programming Problem
}

\author{
O. E. Emam \\ Department of Information \\ Systems, \\ Faculty of Computer Science \\ and Information, Helwan \\ University, P.O. Box 11795, \\ Egypt
}

\author{
E. Fathy \\ Department of Mathematics, \\ Faculty of Science, Helwan \\ University, P.O. Box 11795, \\ Egypt
}

\author{
A. A. Abdullah \\ Department of Information \\ Systems, Higher Institute of \\ Computer Science and \\ Information Systems Fifth \\ Avenue - New Cairo, Egypt
}

\begin{abstract}
The motivation behind this paper is to focus on the solution of a Bi-Level Multi-Objective Large Scale Integer Quadratic Programming (BLMOLSIQP) problem in which all decision parameters in the objective functions are symmetric trapezoidal fuzzy numbers, and has block angular structure of the constraints. The suggested algorithm based on a linear ranking function, weight method, Taylor's series, decomposition algorithm and branch and bound method is to find a compromised solution for the problem under consideration. In addition, the theoretical results are illustrated with the help of a numerical example.
\end{abstract}

\section{Keywords}

Large Scale; Integer Programming; Quadratic Programming; Multi-Objective; Fuzzy Programming; Bi-level programming.

MSC 2010: 90C06; 90C10; 90C20; 90C29; 90C70; 90C99..

\section{INTRODUCTION}

Fuzzy linear programming problems have an essential in fuzzy modeling which can formulate uncertainty in actual environment. Afterwards many authors [1, 2, 3, 4, 5] considered various types of the fuzzy linear programming and propose several approaches for solving these problems.

Bi-level Programming (BLP) is a subset of the multi-level programming problem which identified as a mathematical programming problem that solves decentralized planning problems with two decision makers (DMs) in a two- level or hierarchical organization [6].

The vast majority of the optimization problems emerged in real-world applications incorporates vast numbers of variables and constraints, which are called Large-Scale Programming Problems (LSPP) [7]. One prominent structure of the LSPP is the block angular structure. In this structure, an LSPP is separated into smaller sub-problems which appear together, sharing common resources in the upper-most interconnected constraints $[8,9]$.

Integer Programming (IP) problems are optimization problems that minimize or maximize the objective function taking into consideration the limits of constraints and integer variables. More widely application of integer programming can be used to appropriately describe the decision problems on the management and effective use of resources in engineering technology, business management and other numerous fields [10].

Quadratic programming (QP) is one of the most popular models used in decision-making and in optimization problems
[11]. Quadratic Programming problem aims at minimize (maximize) a quadratic objective function subject to a set of linear constraints. If the coefficients in the objective function are exactly known crisp value, then these models can be solved by classical methods and algorithms.

Emam presented a bi-level integer non-linear programming problem with linear or non-linear constraints [12] and proposed an interactive approach to solve a bi-level integer multi-objective fractional programming problem in [13]. Baky [14] introduced two new algorithms to solve multi-level multi-objective linear programming problems through the fuzzy goal programming approach. The membership functions for the defined fuzzy goals of all objective functions at all levels were developed. Then the fuzzy goal programming approach was used to obtain the satisfactory solution for all decision makers.

Abo-Sinna and Abou-El-Enien [15] extend TOPSIS for solving interactive large scale multiple Objective programming problems involveing fuzzy parameters. These fuzzy parameters are characterized as fuzzy numbers. For such problems, the $\alpha$-Pareto optimality is introduced by extending the ordinary Pareto optimality on the basis of the $\alpha$ Level sets of fuzzy numbers. An interactive fuzzy decision making algorithm for generating $\alpha$-Pareto optimal solution through TOPSIS approach is provided where the decision maker (DM) is asked to specify the degree $\alpha$ and the relative importance of objectives.

Osman et al. [16] presented a method for solving a special class of large scale fuzzy multi-objective integer problems depending on the decomposition algorithm.

Emam et al. [17] solved a Fully Rough Three Level Large Scale Integer Linear Programming (FRTLLSILP) problem, in which all decision parameters and decision variables in the objective functions and the constraints are rough intervals, and have block angular structure of the constraints. The optimal values of decision rough variables are rough integer intervals. The proposed model was based on interval method and slice-sum method in an interactive model to find a compromised solution for (FRTLLSILP).

Emam et al. [18] solved a Fully Fuzzy Multi-Level Linear Programming (FFMLLP) Problem, where all of its decision parameters and variables are fuzzy numbers. An algorithm depending on the fuzzy decision approach and bound and decomposition method to find a fuzzy optimal solution.

This paper is organized as follows: the researches start in Section 2 by formulating the model of (BLMOLSIQP) problem with fuzzy parameters in the objective functions. In 
Section 3 converts the fuzzy number in the objective functions into deterministic form. In Section 4 presents a Taylor series approach for (BLMOLSIQP) problem then converts the quadratic objective functions and using the weight method to transform the objective functions from multi-objective form to single objective form. In section 5, the decomposition method for Bi-level Large Scale Integer Linear Programming (BLLSLIP) problem is presented. An algorithm for solving the proposed problem is suggested in Section 6. In Section 7, a numerical example is provided to describe the developed results. Finally, conclusion and future works are reported in Section 8.

\section{PROBLEM FORMULATION}

A Bi-Level Multi-Objective Large Scale Integer Quadratic Programming (BLMOLSIQP) problem with symmetric trapezoidal fuzzy numbers in the objective functions may be formulated as follows:

\section{[FLDM]}

$\underset{x_{1}, x_{2}}{\operatorname{Max}} F_{1}(x, \tilde{u})=\underset{x_{1}, x_{2}}{\operatorname{Max}}\left[f_{11}\left(x_{j}, \tilde{u}_{11}\right), f_{12}\left(x_{j}, \tilde{u}_{12}\right)\right.$,

$$
\left.\ldots, f_{1 n}\left(x_{j}, \tilde{u}_{1 n}\right)\right]
$$

[SLDM]

Where $x_{3}, \ldots ., x_{m}$ solves

$\operatorname{Max}_{x_{3}, x_{4}} F_{2}(x, \tilde{u})=\operatorname{Max}_{x_{3}, x_{4}}\left[f_{21}\left(x_{j}, \tilde{u}_{21}\right), f_{22}\left(x_{j}, \tilde{u}_{22}\right)\right.$,

$$
\left.\ldots, f_{2 n}\left(x_{j}, \tilde{u}_{2 n}\right)\right]
$$

Subject to

Where $x_{5}, \ldots, x_{m}$ solves

$$
\begin{aligned}
x \in G=\left\{a_{01} x_{1}+a_{02} x_{2}\right. & +a_{0 m} x_{m} \\
d_{1} x_{1} & \leq b_{0}, \\
& \leq b_{1}, \\
& \leq b_{2}, \\
d_{2} x_{2} & \\
& \\
d_{m} x_{m} & \leq b_{m},
\end{aligned}
$$$$
\left.x_{1}, \ldots \ldots, x_{m} \geq \text { and integer }\right\} \text {. }
$$

In the above problem (1) - (3), $x_{j} \in R^{n},(j=1.2, \ldots m)$ be a real vector variables indicating the first decision level's choice and the second decision level's choice. Moreover, the FLDM has $x_{1}, x_{2}$ indicating the first decision level choice, the SLDM have $x_{3}, x_{4}$ indicating the second decision level choice and $\mathrm{n}$ dimensional row vector $(\mathrm{i}=1,2) \quad(\mathrm{k}=1,2, . ., \mathrm{n})$ of fuzzy parameters in the objective functions, $G$ is the large scale linear constraint set where, $b=\left(b_{0}, \ldots ., b_{m}\right)^{\mathrm{T}}$ is $(m+1)$ vector, and $\mathrm{a}_{01}, \ldots, \mathrm{a}_{0 \mathrm{~m}}, \mathrm{~d}_{1}, \ldots, \mathrm{d}_{\mathrm{m}}$ are constants.

\section{Definition 1. [13]}

For any $\left(\mathrm{x}_{1}, \mathrm{x}_{2} \in \mathrm{G}_{1}=\left\{\mathrm{x}_{1}, \mathrm{x}_{2} \mid\left(\mathrm{x}_{1}, \mathrm{x}_{2}, \mathrm{x}_{3}, \ldots, \mathrm{x}_{\mathrm{m}}\right) \in \mathrm{G}\right\}\right)$ given by FLDM, if the decision-making variable $\left(\mathrm{x}_{3}, \mathrm{x}_{4} \in \mathrm{G}_{2}=\left\{\mathrm{x}_{3}, \mathrm{x}_{4} \mid\left(\mathrm{x}_{1}, \mathrm{x}_{2}, \mathrm{x}_{3}, \ldots, \mathrm{x}_{\mathrm{m}}\right) \in \mathrm{G}\right\}\right)$ is the optimal solution of the SLDM, then $\left(\mathrm{x}_{1}, \mathrm{x}_{2}, \mathrm{x}_{3}, \ldots, \mathrm{x}_{\mathrm{m}}\right)$ is a feasible solution of (BLMOLSIQP) problem.

Definition 2. [13]

If $x_{j}^{*} \in R^{n} \quad,(j=1,2, \ldots, m)$ is a feasible solution of the

(BLMOLSIQP) problem; no other feasible solution

$$
x_{j}^{*}
$$

exists, such that $\mathrm{F}_{\mathrm{i}}(\quad) \leq \mathrm{F}_{\mathrm{i}}(\quad \quad),(\mathrm{i}=1,2),(\mathrm{j}=1,2, \ldots, \mathrm{m})$ so is the optimal solution of the (BLMOLSIQP) problem.

\section{RANKING FUNCTION}

To solve (BLMOLSIQP) problem with fuzzy numbers in the objective functions using linear ranking technique that convert fuzzy number form into equivalent deterministic form by using [18], but transformation process needs the following definitions to be known:

Definition 3. [16]

If, then a linear ranking function is defined

as

$$
\Re(\tilde{A})=a+b+\frac{1}{2}(d-c) .
$$

\section{Definition 4. [16]}

Let $\tilde{A}\left(\mathrm{a}_{1}, \mathrm{~b}_{1}, \mathrm{c}_{1}, \mathrm{~d}_{1}\right)$ and $\tilde{B}\left(\mathrm{a}_{2}, \mathrm{~b}_{2}, \mathrm{c}_{2}, \mathrm{~d}_{2}\right)$ is two trapezoidal fuzzy numbers. A convenient method for comparing of the fuzzy numbers is by using of ranking functions. A ranking function is a map from $F(R)$ into the real line. So, the orders on $F(R)$ as follows:

$$
\begin{aligned}
& \text { 1. } \tilde{A} \geq \tilde{B} \text { If and only if } \mathfrak{R}(\tilde{A}) \geq \mathfrak{R}(\tilde{B}) . \\
& \text { 2. } \tilde{A}>\tilde{B} \text { If and only if } \mathfrak{R}(\tilde{A})>\mathfrak{R}(\tilde{B}) . \\
& \text { 3. } \tilde{A}=\tilde{B} \text { If and only if } \mathfrak{R}(\tilde{A})=\mathfrak{R}(\tilde{B}) . \\
& \text { Where } \tilde{A} \text { and } \tilde{B} \text { are in } F(R) .
\end{aligned}
$$

Then the (BLMOLSIQP) problem can be understood as the corresponding deterministic in the objective functions as following:

\section{[FLDM]}

$$
\begin{array}{r}
\operatorname{Max}_{x_{1}, x_{2}} F_{1}(x)=\left[f_{11}(x), f_{12}(x), . ., f_{1 n}(x)\right] \\
\text { Where } x_{3}, \ldots, x_{m} \text { solves }
\end{array}
$$

[SLDM]

$$
\underset{x_{3}, x_{4}}{\operatorname{Max}} F_{2}(x)=\left[f_{21}(x), f_{22}(x), \ldots, f_{2 n}(x)\right](6)
$$$$
\text { Where } x_{5}, \ldots, x_{m} \text { solves }
$$

Subject to

$x \in G$

\section{TAYLOR SERIES APPROACH}

This problem is (BLMOLSIQP) problem and it's difficult to solve this problem using a decomposition algorithm, so the researches transform the objective functions by using $1^{\text {st }}$ order Taylor series polynomial in the following form [19].

$$
\begin{aligned}
& K_{i}(x) \cong F_{i}^{\wedge}(x)=F_{i}\left(x_{j}^{*}\right)+\sum_{j=1}^{m}\left(x_{j}-x_{i j}^{*}\right) \frac{\partial F_{i}\left(x_{j}^{*}\right)}{\partial x_{j}} \text {, Then the } \\
& (j=1,2, \ldots, m),(i=1,2)
\end{aligned}
$$

researches use the weighting method [10] to transform the objective functions in the upper level and lower level from 
multi-objective into single objective the weight of the first objective is greater than the weight of the second objective so the Bi-Level Large Scale Integer Linear Programming (BLLSLIP) problem can be written as:

\section{[FLDM]}

$\underset{x_{1}, x_{2}}{\operatorname{Max}} Q 1(\mathrm{x})$

Where $x_{3}, \ldots, x_{m}$ solves

[SLDM]

$\operatorname{Max} Q 2(\mathrm{x})$

Where $x_{5}, \ldots, x_{m}$ solves

Subject to

$x \in G$.

\section{DECOMPOSITION ALGORITHM FOR BI LEVEL LARGE SCALE LINEAR PROGRAMMING PROBLEM}

The (BLLSLIP) problem is solved by adopting the leaderfollower Stackelberg strategy combine with Dantzig and Wolf decomposition method [9]. First, the optimal solution that is acceptable to the FLDM is obtained using the decomposition method to break the large scale problem into n-sub problems that can be solved directly.

The decomposition technique depends on representing the (BLLSLIP) problem in terms of the extreme points of the sets $d_{j} x_{j} \leq b_{j}, x_{j} \geq 0, j=1,2, . ., m$.To do so, the solution space described by each $d_{j} x_{j} \leq b_{j}, x_{j} \geq 0, j=1,2, . ., m$ must be bounded and closed.

After that by inserting the upper level decision variable to the lower level for him/her to search for the optimal solution using Dantzig and Wolf decomposition method [9], then the decomposition method break the large scale problem into nsub problems that can be solved directly and obtain the optimal solution for his/her problem which is the optimal solution to the (BLLSLIP) problem.

\section{Theorem 1.}

The decomposition algorithm terminates in a finite number of iterations, yielding a solution of the large scale problem.

To prove theorem 1 above, the reader is referred to [9].

\subsection{The First-Level Decision-Maker (FLDM) Problem}

The FLDM problem of the (BLLSLIP) problem is as follows:

$[$ FLDM $]$

$\operatorname{Max} F_{1}(x)=\operatorname{Max} \sum_{j=1}^{m} c_{1 j} x_{j}$

Subject to

$x \in G$.

The FLDM problem use decomposition method [9] to solve his problem. If the FLDM doesn't have an integer optimal, then the FLDM well use branch and abound [10] to get an integer optimal solution.

\subsection{The Second-Level Decision-Maker (SLDM) Problem}

Finally, according to the mechanism of the (BLLSLIP) problem, the First Level variables $x_{1}^{F}, x_{2}^{F}$ should be passed to the Second-Level; so the second -level problem can be written as follows:

$\operatorname{Max}_{2}(x)=\operatorname{Max} \sum_{j=1}^{m} c_{2 j} x_{j}$,

Subject to

$\left(x_{1}^{F}, x_{2}^{F}, \ldots, x_{m}^{F}\right) \in G$.

To obtain the optimal solution of the second -level problem; the SLDM solves his master problem by the decomposition method [9] as the first level, If the SLDM doesn't have an integer optimal, then the FLDM well use branch and abound [10] to get an integer optimal solution.

Now the optimal solution $\left(x_{1}^{F}, x_{2}^{F}, x_{3}^{S}, x_{4}^{S}, x_{5}^{S}, \ldots, x_{m}^{S}\right)$ of the SLDM is the optimal solution of the (BLLSLIP) problem.

\section{AN ALGORITHM FOR SOLVING (BLMOLSIQP) PROBLEM WITH FUZZY NUMBERS}

A solution algorithm to solve (BLMOLSIQP) Problem, in which all decision parameters in the objective functions are symmetric trapezoidal fuzzy numbers, and has block angular structure of the constraints, is described in a series of steps as follows:

Step 1. Formulate the (BLMOLSIQP) Problem.

Step 2. The FLDM and SLDM uses linear ranking technique [18] to compute $\mathfrak{R}(\tilde{A})$ for all the coefficients of the problem (1) - (2), where $\tilde{A}$ is symmetric trapezoidal fuzzy number.

Step 3. Convert from fuzzy number form to equivalent deterministic form by using [18].

Step 4. Formulate the equivalent (BLMOLSIQP) problem with deterministic form in the objective functions (5) (6).

Step 5. Convert (BLMOLSIQP) problem to linear Programming problem by using Taylor series Approach [19] as follow:

$K_{i}(x) \cong F_{i}^{\wedge}(x)=F_{i}\left(x_{j}^{*}\right)+\sum_{j=1}^{m}\left(x_{j}-x_{i j}^{*}\right) \frac{\partial F_{i}\left(x_{j}^{*}\right)}{\partial x_{j}},(j=1,2, \ldots, m),(i=1,2)$ Step

Use weight method [10] to convert from multi objective to single objective.

Step 7. Formulate the (BLLSLIP) problem (8) - (9).

Step 8. Apply the decomposition algorithm [9] to solve the FLDM problem by breaking the large scale problems into $\mathrm{n}$ sub-problems that can be solved directly, then the optimal solution is get (10).

Step 9. If the FLDM gets the optimal solution is an integer, go to Step 11. 
Otherwise, go to Step 10.

Step 10. Use branch and bound method [10] to find integer optimal solution.

Step 11. Set $\left(x_{1}, x_{2}\right)=\left(x_{1}^{F}, x_{2}^{F}\right)$ to the SLDM constraints.

Step 12. Formulate the SLDM problem (11).

Step 13. Apply the decomposition algorithm [9] to solve the SLDM, then the optimal solution is get.

Step 14. If the SLDM gets the optimal solution is an integer, go to Step 16.

Otherwise, go to Step 15.

Step 15. Use branch and bound method [10] to find integer optimal solution.

Step 16. ( $\left.x_{1}^{F}, x_{2}^{F}, x_{3}^{S}, x_{4}^{S}, x_{5}^{S}, \ldots, x_{m}^{S}\right)$ is an optimal solution for (BLMOLSIQP) problem, and then go to Step 17.

Step 17. Stop.

Remark1. For (BLMOLSIQP) problem, the Lingo package is suggested as a basic solution tool.

\section{NUMERICAL EXAMPLE}

To demonstrate the solution for (BLMOLSIQP) with fuzzy numbers, let us consider the following problem:

\section{[FLDM]}

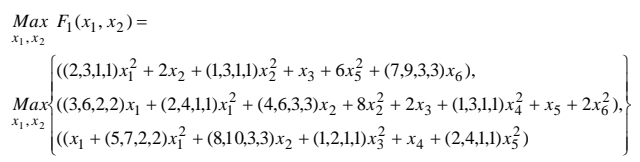

Where $x_{3}, x_{4}, x_{5}, x_{6}$ solves

\section{[SLDM]}

$\underset{x_{3}, x_{4}}{\operatorname{Max}} F_{2}\left(x_{3}, x_{4}\right)=$

$\left(\left(x_{1}+(1,2,1,1) x_{2}^{2}+5 x_{3}^{2}+(3,5,2,2) x_{4}+(4,6,3,3) x_{4}^{2}+3 x_{6}+x_{6}^{2}\right)\right.$,

$\underset{x_{3}, x_{4}}{\operatorname{Max}}\left\{\begin{array}{l}\left.\left(2 x_{1}+(1,3,1,1) x_{1}^{2}+3 x_{2}+4 x_{3}+(7,8,4,4) x_{3}^{2}+(3,5,2,2) x_{4}+(4,6,3,3) x_{4}^{2}+6 x_{5}^{2}+x_{6}\right),\right\} \\ \left(x_{1}^{2}+x_{2}+5 x_{2}^{2}+(10,12,8,8) x_{3}+6 x_{4}+(6,8,4,4) x_{4}^{2}+x_{5}+(1,3,1,1) x_{6}\right)\end{array}\right\}$

Where $x_{5}, x_{6}$ solves

Subject to

$x_{1}+x_{2}+x_{3}+x_{4}+x_{5}+x_{6} \leq 90$,

$2 x_{1}-x_{2} \leq 10$,

$x_{1}+3 x_{2} \leq 35$,

$-x_{3}+x_{4} \leq 50$

$2 x_{5}+x_{6} \leq 40$,

$x_{5}+2 x_{6} \leq 60$

$x_{1}, x_{2}, x_{3}, x_{4}, x_{5}, x_{6} \geq 0$. and integer.

Firstly, Applying ranking function [18] to transform the fuzzy number form in to equivalent crisp form so, the problem reduces to

[FLDM]

$$
\operatorname{Max}_{x_{1}, x_{2}} F_{1}\left(x_{1}, x_{2}\right)=\operatorname{Max}_{x_{1}, x_{2}}\left\{\begin{array}{l}
\left(5 x_{1}^{2}+2 x_{2}+4 x_{2}^{2}+x_{3}+6 x_{5}^{2}+16 x_{6}\right), \\
\left(9 x_{1}+6 x_{1}^{2}+10 x_{2}+8 x_{2}^{2}+2 x_{3}+4 x_{4}^{2}+x_{5}+2 x_{6}^{2}\right), \\
\left(x_{1}+12 x_{1}^{2}+18 x_{2}+3 x_{3}^{2}+x_{4}+6 x_{5}^{2}\right)
\end{array}\right\}
$$

Where $x_{3}, x_{4}, x_{5}, x_{6}$ solves

[SLDM]

$\underset{x_{3}, x_{4}}{\operatorname{Max}} F_{2}\left(x_{3}, x_{4}\right)=\underset{x_{3}, x_{4}}{\operatorname{Max}}\left\{\begin{array}{l}\left(x_{1}+3 x_{2}^{2}+5 x_{3}^{2}+8 x_{4}+10 x_{4}^{2}+3 x_{6}+x_{6}^{2}\right), \\ \left(2 x_{1}+4 x_{1}^{2}+3 x_{2}+4 x_{3}+15 x_{3}^{2}+8 x_{4}+10 x_{4}^{2}+6 x_{5}^{2}+x_{6}\right), \\ \left(x_{1}^{2}+x_{2}+5 x_{2}^{2}+22 x_{3}+6 x_{4}+14 x_{4}^{2}+x_{5}+4 x_{6}\right)\end{array}\right\}$

Where $x_{5}, x_{6}$ solves

Subject to

$x_{1}+x_{2}+x_{3}+x_{4}+x_{5}+x_{6} \leq 90$,

$2 x_{1}-x_{2} \leq 10$,

$x_{1}+3 x_{2} \leq 35$,

$-x_{3}+x_{4} \leq 50$

$2 x_{5}+x_{6} \leq 40$,

$x_{5}+2 x_{6} \leq 60$,

$x_{1}, x_{2}, x_{3}, x_{4}, x_{5}, x_{6} \geq 0$. and integer.

Secondly, applying the first order Taylor series [19] to convert the quadratic objectives functions to linear objectives functions and use the weighting method [10] to convert multi objectives to single objective so the (BLLSLIP) problem is written as following:

[FLDM]

$\operatorname{Max}_{x_{1}, x_{2}} F_{1}\left(x_{1}, x_{2}\right)=\operatorname{Max}_{x_{1}, x_{2}}\left\{\begin{array}{l}\left(10 x_{1}+10 x_{2}+x_{3}+12 x_{5}+16 x_{6}-15\right), \\ \left(21 x_{1}+26 x_{2}+2 x_{3}+8 x_{4}+x_{5}+4 x_{6}-20\right), \\ \left(25 x_{1}+18 x_{2}+6 x_{3}+x_{4}+12 x_{5}-20\right)\end{array}\right\}$

Where $x_{3}, x_{4}, x_{5}, x_{6}$ solves

[SLDM]

$\operatorname{Max}_{x_{3}, x_{4}} F_{2}\left(x_{3}, x_{4}\right)=\operatorname{Max}_{x_{3}, x_{4}}\left\{\begin{array}{l}\left(x_{1}+6 x_{2}+10 x_{3}+28 x_{4}+5 x_{6}-19\right), \\ \left(10 x_{1}+3 x_{2}+34 x_{3}+28 x_{4}+12 x_{5}+x_{6}-35\right), \\ \left(2 x_{1}+11 x_{2}+22 x_{3}+34 x_{4}+x_{5}+4 x_{6}-20\right)\end{array}\right\}$

Where $x_{5}, x_{6}$ solves

Subject to

$x_{1}+x_{2}+x_{3}+x_{4}+x_{5}+x_{6} \leq 90$,

$2 x_{1}-x_{2} \leq 10$,

$x_{1}+3 x_{2} \leq 35$,

$-x_{3}+x_{4} \leq 50$

$2 x_{5}+x_{6} \leq 40$,

$x_{5}+2 x_{6} \leq 60$,

$x_{1}, x_{2}, x_{3}, x_{4}, x_{5}, x_{6} \geq 0$. and integer.

The first level problem is formulated as:

$\operatorname{Max} F_{1}\left(x_{1}, x_{2}\right)=$

$x_{1}, x_{2}$

$\operatorname{Max}\left(17.8 x_{1}+17.2 x_{2}+2.8 x_{3}+2.7 x_{4}+8.7 x_{5}+7.9 x_{6}-18.3\right)$

$x_{1}, x_{2}$ 
Subject to

$x \in G$.

After four iterations the first level decision maker optimal solution is obtained:

$\left(x_{1}^{F}, x_{2}{ }^{F}, x_{3}{ }^{F}, x_{4}{ }^{F}, x_{5}{ }^{F}, x_{6}{ }^{F}\right)=$

$(9.28,8.5,38.8,0,6.66,26.66)$

So, $F_{1}=671.748$

The FLDM use branch and bound to get integer optimal solution then the researches get

$\left(x_{1}{ }^{F}, x_{2}{ }^{F}, x_{3}{ }^{F}, x_{4}{ }^{F}, x_{5}{ }^{F}, x_{6}{ }^{F}\right)=(9,8,38,2,6,26)$.

So, $F_{1}=657.60$

Then take the first level decision maker solution and set $\left(x_{1}{ }^{F}, x_{2}{ }^{F}\right)=(9,8)$ to the second level constraint.

$\operatorname{Max} F_{2}\left(x_{3}, x_{4}\right)=$

$x_{3}, x_{4}$

$\operatorname{Max}\left(4 x_{1}+6.6 x_{2}+20.8 x_{3}+29.8 x_{4}+3.9 x_{5}+3.5 x_{6}-24.1\right)$

$x_{3}, x_{4}$

Subject to

$x \in G$.

The SLDM will repeat the same steps as the first level decision maker until the second level decision maker get the optimal solution so:

$\left(x_{3}{ }^{s}, x_{4}{ }^{s}, x_{5}{ }^{s}, x_{6}{ }^{s}\right)=(11.5,61.5,0,0)$

The SLDM use branch and bound to get integer optimal solution then the researches get

$\left(x_{1}{ }^{F}, x_{2}{ }^{F}, x_{3}{ }^{s}, x_{4}{ }^{s}, x_{5}{ }^{s}, x_{6}{ }^{s}\right)=(9,8,12,61,0,0)$.

So, $F_{1}^{*}=671.748, F_{2}^{*}=2132.100$

\section{SUMMARY AND CONCLUDING REMARKS}

In this paper, the researches presented a bi-level multiobjective large scale integer quadratic programming (BLMOLSIQPP) problem with fuzzy parameters in the objective functions at every level are to be maximized. The solution algorithm was based on a linear ranking function, weight method, Taylor's series, decomposition algorithm and branch and bound method is to find a compromised solution for the problem under consideration. Finally, a numerical example was given to clarify the main results developed in this paper.

However, there are many other aspects, which should by explored and studied in the area of fuzzy large scale optimization such as:

1. Bi-level Multi-objective large scale integer quadratic programming problem with fuzzy parameters in the constraints.

2. Bi-level Multi-objective large scale integer quadratic programming problem with fuzzy parameters in the objective functions and constraints.

3. Multi-level Multi-objective large scale integer quadratic programming problem with fuzzy parameters in the objective functions and constraints.

\section{REFERENCES}

[1] T.H.M.Abou-El-Enien, "On the Solution of a Special Type of Large Scale Integer Linear Vector Optimization Problems with Uncertain Data through TOPSIS Approach", International Journal of Contemporary Mathematical Sciences, 6 (2011) 657 - 669.

[2] S. Barkha, and D. Rajendra, "Optimum Solution of Fuzzy Linear Programming Problem for Trapezoidal Number", VSRD-TNTJ, 3 (7) (2012) 268-276.

[3] N. Mahdavi , and S. H. Nasseri, "Duality in Fuzzy Number Linear Programming by Use of a Certain Linear Ranking Function", Applied Mathematics and Computation, 180 (2006) 206-216.

[4] S. H. Nasseri, E. Adril, A.Yazdani, and R.Zaefarian, "Simplex Method for Solving Linear Programming Problems with Fuzzy Numbers", World Academy of Science, Engineering and Technology, 10.(2005), 285288

[5] E. A. Youness, O. E. Emam, and M. S. Hafez, "Simplex Method for Solving Bi-Level Linear Fractiopnal Integer Programming Problems with Fuzzy Numbers", International Journal of Mathematical Sciences and Engineering Applications. 3 (2013) 351-363.

[6] E. A. Youness, O. E. Emam, M. S. Hafez, "Bi-Level Multi-Objective Fractional Integer Programming", Applied Mathematics \& Information,6 (2014) 28572863.

[7] T. I. Sultan, O. E. Emam and A. A. Abohany, "A Decomposition Algorithm for Solving a Three-level Large Scale Linear Programming Problem", Applied Mathematics and Information Science, 5 (2014) 2217 2223.

[8] M. A. Abo-Sinna and T. H. M. Abou-El-Enien, "An Interactive Algorithm for Large Scale Multiple Objective Programming Problems with Fuzzy Parameters Through Topsis Approach", Yugoslav Journal of Operations Research, 21 (2011) 253-273.

[9] G. Dantzig and P. Wolfe, "The Decomposition Algorithm for Linear Programming", Econometric, 9(4) (1961) $767-778$.

[10] H. T. Taha, "Operation Research-An Introduction", 6th Edition, Mac Milan Publishing Co, New York, 1997.

[11] O. Emam, S. Kholeif and S. Azzam, "A decomposition Algorithm for Solving Stochastic Multi-Level Large Scale Quadratic Programming Problem", Applied Mathematics \& Information Sciences, 9(4), (2015).

[12] O.E. Emam, "A Fuzzy Approach for Bi-Level Integer Nonlinear Programming Problem", Applied Mathematics and Computation, 172 (2006) 62-71.

[13] O.E. Emam, "Interactive Approach to Bi-Level Integer Multi-Objective Fractional Programming Problem", 
Applied Mathematics and Computation, 233 (2013) 1724.

[14] I.A. Baky, "Solving Multi-Level Multi-Objective Linear Programming Problems Through Fuzzy Goal Programming Approach", Applied Mathematical Modeling, 34 (2010) 2377-2387.

[15] M. A. Abo-Sinna and T.H.M.Abou-El-Enien, "An Interactive Algorithm for Large Scale Multiple objective Programming Problems with Fuzzy Parameters through TOPSIS approach", Applied Mathematics and Computation, 177 (2006)515-527.

[16] M.S. Osman, O.M. Saad, and A.G. Hasan , "Solving Special Class of Large Scale Fuzzy Multi Objective Integer Linear Programming Problems", Fuzzy Sets and Systems, 107 (1999) 289-297.
[17] O. E. Emam, E. Fathy and A. A. Abohany, "An Interactive Model for Fully Rough Three Level Large Scale Integer Linear Programming Problem", International Journal of Computer Applications, 155 (11) (2016) 1-12.

[18] O. E. Emam, E. Fathy and M. A. Helmy, "Fully Fuzzy Multi-Level Linear Programming Problem", International Journal of Computer Applications 155 (7) (2016) 18-26.

[19] O. M. Saad, T. R. Mohamed, M. K. Alshafae, E. F. Abdellah, "Taylor Series Approach for Solving ChanceConstrain Multi Objective Integer Linear Fractional Programming Problem", International Journal of Mathematical Archive, 3 (2012) 18 - 23. 